Referencia para citar este artículo: Moreno-Roldán, M. R., Agudelo-Bedoya, M. E., \& Alzate-Pulgarín, V. (2018). Voces a escuchar en el cuidado: ¿qué dicen los niños y las niñas? Revista Latinoamericana de Ciencias Sociales, Niñez y Juventud, 16(1), 227-237. doi:10.11600/1692715x.16113

\title{
Voces a escuchar en el cuidado: ¿qué dicen los niños y las niñas?*
}

\author{
MARGARITA Rosa MORENO-ROLDÁN ${ }^{* *}$ \\ Profesora Universidad Pontificia Bolivariana, Colombia. \\ MARÍA EUGENIA AGUDELO-BEDOYA ${ }^{* * *}$
Profesora Universidad Pontificia Bolivariana, Colombia.

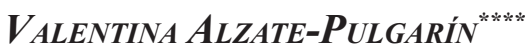 \\ Estudiante de Trabajo Social, Colombia.
}

\section{Artículo recibido en junio 6 de 2017; artículo aceptado en septiembre 22 de 2017 (Eds.)}

- Resumen (analítico): este artículo presenta los resultados derivados de un estudio cualitativo en la ciudad de Medellín, especificamente de seis grupos focales realizados con niños y niñas entre los cinco y doce años de edad. El principal propósito fue comprender mediante las voces de los y las participantes, el significado del cuidado, el uso de las redes y las diferencias de cuidado según el género. En los hallazgos, si bien, surge la participación del hombre - padre, primo, tio o hermano-, en el cuidado prevalece la figura femenina - madre, tía, hermana o abuela-. La preferencia entre un cuidador/cuidadora $u$ otro/otra está relacionada con las actividades que ellos y ellas realizan con los niños y niñas y el trato que estos y estas reciban. Se concluye que el cuidado integra aspectos económicos, materiales y afectivos, siendo los últimos los más relevantes desde sus relatos.

Palabras clave: cuidado de niño, infancia (Tesauro de Ciencias Sociales de la Unesco); género (Tesauro Colombiano en Familia y Género).

\section{Voices to listen to about child care: What do children say?}

- Abstract (analytical): This article presents the results of a qualitative research study conducted in the city of Medellin based on six focus groups involving boys and girls aged 5 to 12 years old. The main objective of the study was, based on the information provided by boys and girls, to comprehend the meaning of child care, the use of support networks and differences in child care based on a child's gender. The findings of the study suggest that, though male relatives play a role in care (father, uncle,

\footnotetext{
* Este artículo de investigación científica y tecnológica. Área de conocimiento: Ciencias Sociales. Subárea: Ciencias Sociales, Interdisciplinaria. Se basa en la investigación "La organización social del cuidado de niños y niñas menores de 12 años en grupos familiares: caso Medellín", financiada por la Universidad Pontificia Bolivariana (UPB) y la Fundación Bien Humano entre el 01 de octubre de 2015 y el 01 abril de 2017 con número de Radicado 500B-10/15-15. Está integrada a un Macroproyecto efectuado en cinco ciudades de Colombia. En Medellín, fue ejecutada por los Grupos de investigación Familia y Derecho de la UPB; contó con la participación de estudiantes de pregrado de Trabajo Social y de la Especialización en Familia; se llevaron a cabo 70 entrevistas a cuidadores y cuidadoras de todos los estratos socioeconómicos y seis grupos focales. Tipo cualitativo, enfoque hermenéutico.

** Trabajadora Social de la Universidad de Antioquia y Magíster en Terapia Familiar de la Universidad Pontificia Bolivariana de Medellín, Colombia. Docente de la Facultad de Trabajo Social de la Universidad Pontificia Bolivariana. Orcid: 0000-0003-1655-1299. Correo electrónico: margaritarosa.moreno@upb.edu.co

*** Trabajadora Social y Magíster en Terapia Familiar de la Universidad Pontificia Bolivariana de Medellín, Colombia; Docente interno de la Facultad de Trabajo Social. Orcid: 0000-0001-6637-2841. Índice H5: 4. Correo electrónico: maria.agudelo@upb.edu.co

**** Estudiante de Trabajo Social de la Universidad Pontificia Bolivariana de Medellín, Colombia. Orcid. 0000-0002-1250-6979. Correo electrónico: valee.94@hotmail.es
} 
cousin or brother), the female figure prevails (mother, aunt, sister or grandmother). The preference of children for one caregiver over another is related to the type of activities they engage in with that person and how they feel that they are treated. In conclusion, child care consists of economic, material and emotional aspects, with the latter being the most relevant according to the children that participated in the study.

Key words: childcare, childhood (Unesco Social Sciences Thesaurus), gender (Colombian Thesaurus on Family and Gender).

\section{Vozes para escutar no cuidado: o que dizem meninos e meninas?}

- Resumo (analítico): Este artigo apresenta os resultados derivados de um estudo qualitativo na cidade de Medellín, especificamente de seis grupos focais realizados com meninos e meninas entre os cinco e doze anos de idade. O propósito principal foi compreender por meio das vozes dos participantes, os significados do cuidado, o uso das redes e as diferenças do cuidado segundo o gênero. Ainda, que nos resultados aparece a participação do homem — pai, primo, tio ou irmão-no cuidado prevalece a figura feminina — mãe, tia, irmã ou avó-como cuidadora. A preferência entre um cuidador ou outro está relacionada com as atividades realizadas com as crianças e o tratamento que estes recebam. Conclui-se então, que o cuidado considera aspectos econômicos, materiais $e$ afetivos, sendo esses últimos os mais relevantes desde seus depoimentos.

Palavras-chave: cuidado das crianças, infância (Thesaurus de Ciências Sociais da Unesco); gênero (Thesaurus Colombiano em Família e Gênero).

-1. El cuidado: lo amplio del concepto, lo inacabado del tema. -2. Metodología. -3. Relatos sobre el cuidado: sentires de niños y niñas. -4. Conclusiones. -Lista de referencias.

\section{El cuidado: lo amplio del concepto, lo inacabado del tema}

El cuidado ha sido estudiado desde disciplinas como la sociología, la economía, el derecho, la psicología, la enfermería y el trabajo social. Como lo sostienen Micolta, Paz y Jiménez (2016), en el desarrollo de la acción de cuidar hay dos aspectos fundamentales: uno tiene que ver con la persona que brinda los cuidados y la que los recibe, mientras que el otro se refiere al intercambio de afectos, bienes materiales y económicos, y a la existencia de un universo simbólico. En ello coinciden Flores y Tena (2014) quienes hablan de la dimensión relacional de los cuidados y consideran que está definida por la dependencia, dado que en dicha situación, una persona es tributaria de otra para su cuidado y bienestar, ya sea que los cuidados se efectúen dentro o fuera del ámbito familiar.

De acuerdo con Aguirre (2008), el cuidado se constituye en aspecto fundamental que trasciende lo establecido jurídicamente e incluye la construcción y el mantenimiento de vínculos; no obstante, socialmente no hay un reconocimiento de lo que implica, pues se visualiza como una actividad femenina que en la mayoría de los casos no se remunera.

Planteamientos acerca del cuidado, así como observaciones cotidianas sobre lo que ocurre en nuestro país en esta materia, generaron inquietudes en distintas regiones de Colombia acerca de cómo se organizan las familias en torno a este y su relación con el Estado, el mercado y las redes de apoyo. En la revisión de investigaciones que abordaran el tema del cuidado a partir del 2005 se identificaron estudios referidos principalmente al cuidado de personas adultas mayores, con discapacidad y con enfermedades de alto riesgo, las cuales aportaron elementos conceptuales y contextuales para la comprensión del cuidado y afianzaron la importancia de realizar un macroproyecto en el que la perspectiva socioeconómica y de género fueran transversales en el análisis (Barrera et al., 2015). En Medellín, se logró escuchar la voz de quienes son cuidados: los niños y las niñas, así como rescatar sus relatos y sentires acerca del cuidado que reciben. 
Este artículo pretende aportar a la comprensión del concepto de cuidado al presentar la perspectiva de quienes son cuidados/ cuidadas y no de quienes cuidan; intentará responder algunos interrogantes: ¿cuáles son los significados que los niños y las niñas le otorgan al cuidado?, ¿qué sentimientos genera en ellos y ellas?, ¿son todos de satisfacción o existen algunos de insatisfacción?, ¿sus cuidadores y cuidadoras acuden a alguna red para apoyarse en caso de requerirlo?, ¿para los niños y las niñas existen diferencias entre el cuidado de un hombre y el cuidado de una mujer?, ¿hay alguna preferencia entre uno u otro género? Acudiendo a lo que dice Porkinghorne (citado por Agudelo-Bedoya, \& Estrada, 2011): "hasta la más humilde de las narraciones es siempre más que una serie cronológica de acontecimientos: es la recopilación de los acontecimientos para formar una historia con sentido" (p. 30).

En un primer momento se presentan los aspectos metodológicos que permitieron el desarrollo de la investigación; en un segundo momento se desarrollan los hallazgos y la discusión a partir de las categorías: género y cuidado; significado del cuidado y redes de cuidado; y, finalmente, en un tercer momento se presentan las conclusiones del estudio. Como lo proponen Sánchez y Palacio (2013), la investigación en este campo debe conducir a que la familia sea un escenario político y democrático en el que imperen prácticas de cuidado incluyentes de todos los integrantes de la familia, contando con el compromiso de los sectores público, privado y del Estado.

\section{Metodología}

La investigación fue de tipo cualitativo, desde el cual, según Galeano (2014), la realidad se concibe "como resultado de un proceso histórico de construcción a partir de las lógicas de sus protagonistas, con una óptica interna y rescatando su diversidad y particularidad" (p.18). De esta manera, se entiende que no existe una única forma de cuidar a los niños y las niñas ni de vivir el cuidado que reciben. El enfoque metodológico fue el hermenéutico; se buscó comprender las formas en que se organizan los grupos familiares para el cuidado mediante

Rev.latinoam.cienc.soc.niñez juv 16 (1): 227-237, 2018 http://revistalatinoamericanaumanizales.cinde.org.co doi: $10.11600 / 1692715 \times .16113$ la articulación con el Estado, el mercado, las redes vecinales y las $\mathrm{ONG}$, según el género y el estrato socioeconómico. Además, se pretendió reconocer los significados que los niños y las niñas le otorgan al cuidado y aquello que les gustaría modificar de aquel.

El estudio hace parte de un macroproyecto que vinculó universidades ${ }^{1}$ de cinco ciudades del país, a saber: Manizales, Cali, Bogotá, Cartagena y Medellín. En esta última, fue liderado por el grupo de investigación Familia y Derecho de la Universidad Pontificia Bolivariana y por la Fundación Bien Humano, en colaboración con estudiantes de pregrado de la Facultad de Trabajo Social y de la Especialización en Familia. Respecto a las técnicas utilizadas, se efectuaron 70 entrevistas semiestructuradas a cuidadores y cuidadoras principales, así como seis grupos focales con niños y niñas. El estudio se llevó a cabo en dos fases: en el 2015 se desarrollaron las primeras 30 entrevistas y la totalidad de los grupos focales; todas las personas participantes estaban ubicadas en los estratos socioeconómicos ${ }^{2} 1,2$ y 3 y pertenecían a las instituciones que conforman la Alianza para la infancia en Medellín; estas son: Fundación las Golondrinas, Fundación Carla Cristina, Comité Privado de Asistencia a la Niñez (PAN), Fundación Ximena Rico, Jardín infantil la Visitación y Fundación de Atención a la Niñez (FAN). Por su parte, en el 2016 se realizaron 40 entrevistas más con cuidadores y cuidadoras ubicadas mediante la técnica de bola de nieve, en todos los estratos socioeconómicos con énfasis en el 4, 5 y 6.

A continuación se describen solo los aspectos metodológicos referidos a los grupos focales. Se conformó un grupo focal en cada una de las instituciones mencionadas; tres de ellos integrados por niños y niñas de cino y seis años de edad; los otros tres, entre siete y doce años, para un total de 27 participantes. En cada grupo se contó con el apoyo de una docente

1 Universidad Nacional de Colombia y Pontifica Universidad Javeriana, ambas en la ciudad de Bogotá; Universidad de Cartagena, Universidad de Caldas, Universidad del Valle y Universidad Pontificia Bolivariana de Medellín.

2 La estratificación socioeconómica en Colombia identifica geográficamente sectores con distintas características en cuanto a condiciones de los inmuebles, dotación de servicios públicos, de vías y de recursos en educación, salud, recreación, entre otros. 
de la institución, quien moderó la actividad y aportó su formación y experiencia pedagógica para el trabajo con población infantil. Se construyó una guía de entrevista grupal. Dado el momento vital de los y las participantes, en el que se reconoce que su nivel de atención y concentración se mantiene durante periodos cortos de tiempo, los grupos se efectuaron en dos sesiones separadas por un receso. En ambas se utilizó una estrategia lúdica planeada previamente que consistía en un cuento, una canción o una carrera de observación; con los niños y las niñas mayores de siete años se llevó a cabo el juego "el pregunturístico"3.

En relación con los principios éticos del estudio, se comunicó a los cuidadores y cuidadoras los propósitos, quienes firmaron el consentimiento informado y autorizaron la participación de los niños y las niñas, así como la grabación en video de cada encuentro. A los niños y las niñas también se les explicó la actividad y se les solicitó su firma o su huella como muestra de que aceptaban participar. Otras consideraciones éticas contempladas fueron el respeto, la confidencialidad y el retorno social de la información obtenida.

Para el procesamiento de la información, se transcribió el contenido de los grupos focales y se construyeron códigos ${ }^{4}$ para cada participante, en aras de rescatar lo particular de las experiencias frente al cuidado y de esta forma conservar la confidencialidad. También se elaboraron memos analíticos para cada grupo focal; toda la información generada se ingresó en una matriz categorial que permitió hacer un análisis intra e intertextual (Jiménez $\&$ Torres, 2006). Finalmente, se dio paso a la elaboración de los resultados de investigación, la contrastación teórica y la generación de conclusiones.

3 Juego tipo escalera que lleva a participantes a ganar o perder casillas, según el punto que le corresponda al tirar el dado. El punto de meta es igualmente salida y llegada. Se elaboró con preguntas referidas al cuidado. Diseño y creación de Juan L. E.

4 Los códigos se elaboraron de la siguiente manera: se ubicaron letras y números según el grupo focal en el que estaba el niño o la niña (GF1, GF2, GF3, GF4, GF5, GF6), luego la inicial del género (F: femenino; M: masculino); el rango de edad del niño o niña ( 0 a 5 años PI -primera infancia-; 6 a 12 años $\mathrm{N}$-niñez-); y, finalmente, el número del participante (del 1 al 27).

\section{Relatos sobre el cuidado: sentires de niños y niñas}

El siguiente apartado presenta los hallazgos y el análisis de las categorías contempladas en los grupos focales, a saber: diferencias o desigualdades de género en el cuidado, significado del cuidado y redes de apoyo. De esta manera, se plantea cómo observan los niños y las niñas el cuidado que reciben de hombres o mujeres, sus sentires en cuanto al cuidado y sus reacciones frente a las redes de apoyo a las que acuden sus cuidadores y cuidadoras.

3.1. "A mí me gusta que los hombres y las mujeres me cuiden porque son divertidos" (GF3FN26)

En este estudio la categoría de género es transversal en tanto posibilita reflexionar sobre las relaciones construidas entre lo femenino y lo masculino y, en especial, sobre los discursos y las prácticas culturales en torno al cuidado y las diferencias de género que se presentan. En un primer momento, se describen las tendencias de un género con respecto al otro en el cuidado; en un segundo momento, se nombran las percepciones de los participantes frente al cuidado de uno u otro género; $y$, finalmente, presentan las similitudes o las diferencias del cuidado según el género.

Según Carrasco (2006), "tradicionalmente el cuidado no se ha considerado como una responsabilidad social sino como un tema privado $\mathrm{y}$, en especial, como un asunto de mujeres" (p. 41). De acuerdo con la autora, en la actualidad algunas mujeres asumen el trabajo de cuidado y, a la vez, participan en otros ámbitos sociales; ello les exige moverse en un continuo ir y venir entre los distintos espacios de relaciones, así como también les genera una sobrecarga de actividades $\mathrm{y}$, de forma paralela, minimiza la participación de los hombres en el cuidado. Asimismo, Martínez (2005, citada por Flores \& Tena, 2014) sostiene que en América Latina, donde se ha impuesto regímenes de bienestar - contexto que incluye a Colombia-, la constante ha sido la feminización de las responsabilidades de cuidado no remuneradas; lo anterior se articula 
con una ideología familista de fundamentación judeocristiana que valora e impone la familia nuclear como modelo cultural y exalta la virtud femenina como cuidadora.

Acerca de quienes cuidan a los niños y las niñas, en la investigación se halló que prevalece el cuidado como una actividad efectuada por mujeres. En este sentido, la convergencia es que el cuidado es ejercido por figuras femeninas, ya sean madres, tías, abuelas o hermanas, quienes se encargan de las actividades de alimentación, acompañamiento escolar y recreación. Lo anterior coincide con lo que plantean Flores y Tena (2014), quienes afimar que "en el régimen familista, la figura de la madre es aún el símbolo de la estabilidad social" (p. 34). Entrevistador (en adelante E.): "¿Quién te cuida en tu familia?" Entrevistado (en adelante EN.): "Mi hermanita la mayor" (GF1FN7). E.: “¿Quién te cuida en tu familia?" EN.: "Mi mamá, porque ella es la que me alista para ir al colegio, me alimenta y me cuida" (GF5MN22).

Aun así, existe un elemento en común entre niños y niñas, quienes manifiestan que algunos de sus cuidadores son hombres, entre los que se encuentran sus papás, tíos, hermanos, primos y padrastros. Estos participan en actividades recreativas principalmente y en algunos casos en apoyo escolar u otras labores complementarias a las efectuadas por las mujeres. E.: "¿Quién te cuida en la casa?" EN.: "Mi padrastro, el que me cuida casi más es como mi padrastro (GF1MN8)." E.: "A ti ¿te cuidan hombres?" EN.: "Sí, mi papá y mi tío.” E: “¿Y cómo te cuidan tu tío y tu papa?" EN.: "Me hacen el almuerzo y me cuidan mientras que llega mi mamá" (GF4FPI17).

Estos relatos evidencian una participación del hombre en el cuidado que, si bien es menor que la mujer, podría articularse con los planteamientos de Salas (2013) quien, retomando a Geldres, et. al., 2013, menciona otras formas de concebir la paternidad: "Paternar es el nuevo verbo que hace referencia a los hombres como cuidadores de vida (...) implica nuevas actitudes y conductas del hombre frente a sus tareas de reproducción y crianza" (p. 12).

Respecto a las percepciones que tienen los y las participantes frente al cuidado de uno $\mathrm{u}$ otro género, se encuentra que estas varían según la preferencia de las actividades que comparten con el cuidador o la cuidadora y el trato que reciben. Quienes se inclinan por el cuidado de una figura femenina, lo hacen por tres razones: la dedicación del tiempo, las expresiones de afecto y la realización de actividades que consideran son propias de las mujeres. Existe una tendencia a que las niñas se inclinen por el cuidado femenino y los niños por el masculino, si bien hay excepciones que refieren preferencias por el género contrario, en especial del hombre hacia la mujer; en estos casos se visualiza que el criterio principalmente es el trato que les proporcionan o la no participación masculina. E. 1: "¿Quién te encanta que te cuide?" EN.: "Mi mamá, porque ella es muy cariñosa" (GF2MPI1).

En relación con el cuidado por parte del hombre, las narrativas de los niños y las niñas refieren momentos de diversión que comparten con ellos. E1.: "¿Quién te encanta que te cuide?" EN.: "Emm, mi primito (...) él juega conmigo en el celular y tenemos un juego; yo tengo una tablet con el juego de fútbol y él también y me deja jugar con su celular porque a veces mi tablet se está cargando" (GF2MPI3). Se evidencia participación en actividades como ir al parque, jugar, recreación, las cuales son definidas por los niños y las niñas como cuidado. Asimismo, la preferencia por el cuidado del hombre también se alude a la realización de actividades que implican un costo económico. E.: "¿A ti te gusta que te cuiden más las mujeres o los hombres?" EN.: "Los hombres, porque mi papá siempre me lleva al parque y me invita a comer" (GF4FPI14). Esto concuerda con las ideas de Triana, Ávila y Malagón (2010) respecto a que "el rol del padre ha ido cambiando a lo largo de los años y ha estado ligado a los cambios y transformaciones por los cuales ha atravesado la familia" ( $p$. 398). De ahí la insistencia de investigadoras como Cafaro y Espasandin (2015) en "seguir profundizando sobre el enfoque relacional de género y específicamente, incluir el análisis sobre masculinidades" (p. 128) como parte de las estrategias al abordar la comprensión de la organización social del cuidado.

En los relatos en que se manifiestan diferencias en el cuidado entre hombres $y$ 
mujeres se identifican discursos tradicionales frente a lo femenino y lo masculino que atribuyen a las mujeres características desde la ternura y la delicadeza, mientras que a los hombres desde la brusquedad y la agresividad. En el caso de las niñas: EN.: "Sí, porque las mujeres son más dulces y los hombres son muy agresivos" (GF5FN23). En el caso de los niños en algunos relatos también se evidencia una asimilación de los estereotipos de género: E.: “¿Te gusta que te cuiden más los hombres o las mujeres?" EN.: "Los hombres, porque son fuertes y grandes como yo" (GF4MPI16). Además, se observa una articulación de las actividades de cuidado en relación con lo validado culturalmente para uno y otro género. En este encuentro con las voces de niños y niñas se vislumbra lo que Sánchez y Palacio (2013) denotan como "los entramados culturales y emocionales de un discurso familístico que hace necesario confrontar y superar los esencialismos" (p. 37), los cuales tienden a transmitirse entre generaciones, a la vez que justifican las desigualdades de género; estas últimas también se hacen evidentes en lo que al ámbito del cuidado se refiere.

E. 1: “¿Por qué te gusta que te cuiden más las mujeres?" EN.: "Porque ellas me pueden poner lo que yo quiera, me pueden poner vestidos, tacones, aplanchar el pelo, collares y me pueden maquillar. Y mi mamá en el cumpleaños me va poner un vestido, me va a maquillar, aplanchar el pelo y me va a poner una corona, con eeehhh, color piel con unos brillantes" (GF4FPI14).

Como un aspecto particular en los hallazgos se encuentra un caso en el que el niño considera igual el cuidado de un hombre frente al de una mujer E.: "¿Hay alguna diferencia en que te cuide un hombre o que te cuide una mujer?" EN.: "No, porque el hombre debe querer igual que la mujer." (GF5MN24). Se observa una confluencia entre las concepciones tradicionales y las emergentes; en las primeras se evidencia una descripción del comportamiento de cada género y de las formas en cómo se asume el cuidado que ratifican los patrones culturales; en las segundas se encuentran discursos que desdibujan estas diferencias y presentan el cuidado como algo que trasciende el género y lo ubican como un asunto vital que tanto unos y otras deben realizar.

\subsection{E.: "¿Cómo te gusta que te cuiden?" EN.: "Con mucho amor" (GF5FN25)}

En cuanto a la categoría de significados del cuidado, en esta se describen las definiciones y sentires de los niños y las niñas acerca del cuidado que reciben. Carrasco (2006) señala que cuidar implica una responsabilidad $\mathrm{y}$ requiere de una disposición del cuidador $\mathrm{o}$ cuidadora para atender de manera integral las necesidades de quien esté a su cargo. El cuidado vincula asuntos emocionales, relacionales, afectivos e instrumentales que superan la posibilidad de ser cuantificados y todos estos son aspectos absolutamente necesarios para el cuidado de niños y niñas, que configuran la dimensión subjetiva del cuidado, la cual tiende a permanecer oculta ante la sociedad.

En esta misma línea, para Letablier (citado en Aguirre, 2003) el cuidado hace alusión a la práctica, remunerada o no, mediante la cual se ayuda al niño o niña en el logro de su bienestar y en el desarrollo de su vida cotidiana. Se distinguen tres dimensiones del cuidado, las cuales permiten comprender lo amplio del concepto. La primera, la dimensión material, implica un trabajo; la segunda, económica, supone un costo; y la tercera involucra lo afectivo, lo emotivo y lo sentimental. Unido esto a lo que plantean Agudelo-Bedoya y Estrada (2013), en relación a que "como los valores, normas, creencias, actitudes, tradiciones y prácticas varían de un grupo cultural a otro, así mismo varía la construcción social del conocimiento" (p. 19). En esta investigación se halló que algunas definiciones de cuidado de los niños y las niñas refieren una de estas dimensiones.

Desde la dimensión material, los niños y las niñas relacionan el cuidado con la acción de fomentar espacios con ellos $\mathrm{y}$ ellas; comprarles algún objeto que les genere diversión o entretenimiento. Todo esto significa satisfacción en el cuidado que les brindan y, además, representa el esfuerzo del cuidador o cuidadora para obtener el dinero y el tiempo que les permita realizar estas actividades. E.: "¿Quién te cuida en tu familia?" EN.: "Mi mamá.” E.: “¿Por qué?” EN.: "Porque ella es la que trabaja para mí, para darme la comida a mí” 
(GF5MN24). Aguirre (2003) da cuenta de esta implicación del cuidado material en cuanto a la disposición que tienen las personas adultas para la realización de actividades de cuidado.

La segunda forma de entender el cuidado es denominada por Aguirre (2003) como la dimensión afectiva, emotiva y sentimental. En este estudio fue la más mencionada. El cuidado para los niños y las niñas está significado en la recreación, el juego, el amor y el acompañamiento. E.: “QQué haces en tu casa?” EN.: "Jugar, saltar e ir a charco" E.: "¿Y cómo te sientes cuando te llevan a los charcos?" EN.: "Feliz porque a mí me gusta mucho ir a pasear" (GF3FN26).

La tercera dimensión desde la cual se entiende el cuidado es la económica. Las expresiones de los niños y las niñas evidencian que este les genera un costo a las familias; según Esquivel, Faur y Jelin (2011), el tiempo y el dinero que madres y padres destinan para satisfacer las necesidades físicas y emocionales de los niños y niñas lo dejan de emplear en otros usos, por tanto, suponen "costos".

Converge en los relatos la interconexión de las tres dimensiones del cuidado, de tal forma que para algunos niños y niñas, la acción material como el esfuerzo de su cuidador por trabajar se convierte en una forma de cuidado afectivo. A su vez, las actividades que refieren con sus cuidadores y cuidadoras comprenden espacios de recreación como ir al parque, comer helado, ir al zoológico, a centros comerciales $\mathrm{u}$ otros lugares, las cuales evidencian el costo económico y material que le representan a la familia y, a la vez, favorecen el fortalecimiento del vínculo afectivo E.: "¿Dónde te gusta que te lleven?" EN.: "Donde están los elefantes." E.: “¿Y a dónde más?" EN.: "Al centro, a los centros comerciales (...) allá como cositas" (GF2FPI2). Lo anterior encuentra concordancia con lo que plantea Carrasco (2006): "hay que recordar que el trabajo de cuidado, además de que posiblemente se concrete en una determinada actividad, implica importantes dosis de relación, atención psicológica y sostén emocional y afectivo" (p. 52).

Es así como la práctica de cuidar exige un vínculo empático entre quien cuida y quienes son cuidados/cuidadas. En esta investigación los niños y las niñas nombran la empatía con su cuidador y cuidadora en las formas de trato que estos les brindan, desde la protección, amor y acompañamiento; todo lo cual se entendería como el reconocimiento del otro/otra como un sujeto de derechos, donde la existencia de unos y otras, converge y fluye, dando sentido a la dimensión afectiva del cuidado.

Cabe resaltar que existe una tendencia a que los niños y las niñas asocien la satisfacción en el cuidado con el juego, la alimentación, la recreación y la compañía. E.: "Y ¿cómo te cuidan donde tu tía?" EN.: "Voy a piscina, compramos comida de la calle y juego" (GF5FN25). La edad está articulada con esta perspectiva pues en la infancia estos espacios se constituyen en una forma importante en la que los niños y las niñas interactúan entre sí, con personas adultas y conocen su mundo y su alrededor. Esto es muy importante si se tiene en cuenta que "los niños y niñas [que] distinguen un elevado nivel de apoyo de parte de sus padres y madres, son más adaptativos y reducen los efectos negativos de los estresores sobre la salud mental" (GómezUrrutia \& Jiménez-Figueroa, 2015, p. 143).

Un hallazgo importante consiste en el motivo de insatisfacción frente al cuidado en los relatos de niños y niñas. Aparece como tendencia la inconformidad con el castigo físico que ejercen los cuidadores y cuidadoras: "Mi mamá un día me pegó en la pierna y se me volvió todo eso morado.” E. 1: “¿Cómo te cuida la mamá o la mamita cuando estás en la casa?" EN.: “¡Disque pegándome!” (GF2MPI1). EN.: "No me gusta que me peguen, tampoco que me regañen" (GF4FPI14). De esta manera, la expresión de malestar por la práctica del castigo físico es notoria; no se presenta ningún otro motivo de inconformidad frente al cuidado.

En relación con lo anterior, Salas (2013) plantea que "criar, formar y educar para la libertad y la autonomía, implica dejar de lado concepciones, estilos y creencias jerárquicas y adultocéntricas. Las prácticas violentas, el autoritarismo (...) son contrarias al derecho del libre desarrollo de la personalidad" (p. 8). $\mathrm{Si}$ bien existen debates en torno a prácticas tradicionales relacionadas con el castigo físico, en algunos espacios familiares aún están arraigadas como "mecanismos de educación", 
dejando de lado otras formas de crianza que potencialicen el ejemplo y el diálogo, erradiquen esquemas autoritarios que usan la imposición y la fuerza y se acuda a la educación y al cuidado basados en el respeto por todos los integrantes del grupo familiar, independiente de su edad y su rol. Asimismo, el concepto de "crianza humanizada" aporta a la discusión, la cual es descrita por Posada-Díaz, GómezRamírez y Ramírez-Gómez (2008) como el arte de cuidar y criar niños, niñas y adolescentes a partir del establecimiento de vínculos afectivos que posibilitan la construcción de aprendizajes significativos para todas y todos, incluidos los adultos; se relaciona también con el proceso de educación, acompañamiento, orientación y respaldo.

En síntesis, la manera como los niños y las niñas significan el cuidado evidencia un reconocimiento al esfuerzo que realizan los adultos y las adultas para proporcionarlo. Lo que se ubica es que, si bien para ellos y ellas el cuidar puede estar representado en la alimentación, la asistencia al médico o el ingreso a espacios educativos, para los niños y las niñas lo más relevante resultan ser aquellos espacios compartidos con sus cuidadores y cuidadoras que están mediados por el afecto, el juego y la recreación.

3.3. Redes de cuidado. Izquierdo (citado por Garcés \& Chamorro, 2011) las nombra como "figuras subsidiarias" y refiere que son "aquellas que pueden reemplazar al padre o al principal cuidador durante sus ausencias, procurándole al niño, los cuidados que éste necesita y una base segura para la exploración" (p. 41). Es así como el cuidado ha sido fortalecido mediante la creación de dichas redes de apoyo que se encargan de colaborar con los cuidadores principales en los momentos en que se hace necesaria la presencia de otras personas, bien sea de manera retribuida o no en términos económicos. En esta investigación se identifican redes familiares (representadas por abuelos, abuelas, tíos, tías, hermanos y hermanas), vecinales (es decir, personas externas a la familia que tienen cercanía al cuidador o cuidadora principal) e institucionales (que son las entidades públicas y privadas que prestan los servicios de atención al cuidado).

Los niños y las niñas describen ventajas y tensiones que desde su perspectiva se dan cuando se acude a alguna de estas redes (familiar, vecinal, institucional). Es importante mencionar que no indican preferencias de una red con respecto a otra; lo que se observa es que predomina el uso de las redes familiar e institucional sobre la vecinal. De acuerdo con lo anterior, la tendencia es señalar a la familia como la red de apoyo para que padres y madres cumplan con sus labores u oficios fuera de la residencia. E.: "Cuando tu papá y tu mamá están trabajando, ¿quién te cuida?" EN.: "Mi abuelita porque mi papá no puede" (GF1FN11).

Estas redes se constituyen en una ayuda ante las obligaciones laborales o frente a la realización de otras actividades; de ahí que la decisión sobre el cuidado de los niños y las niñas se relacione con la participación laboral, pues los padres y las madres deben delegar el cuidado a personas secundarias. E.: “¿Quién te cuida además de la mamá y del papá si ellos no pueden hacerlo?" EN.: "Mi mamita me cuida mucho porque no está enferma, porque me da el desayuno tan rico" (GF2FPI4). Hay que mencionar, además, que algunas veces los padres y las madres acuden a esta red dado que deben realizar otras actividades. E.: "¿Quién te cuida en la casa cuando la mamá se va para el centro?" EN.: "Me cuida mi mamita (...) si ella no está, me recoge mi tío, y si mi tío no está, entonces me recoge mi mamá" (GF2MPI). Esto concuerda con lo que expresa Zibecchi (2014), al indicar que las investigaciones en América Latina dan cuenta de que, en la región, el trabajo de cuidado es asumido mayormente por las familias, y dentro de los hogares, por las mujeres; ello está estrechamente ligado con la "ideologización del trabajo doméstico".

Según sus relatos, las actividades de cuidado que adquieren un significado relevante en la red familiar giran en torno al juego. En particular, se evidenciaron tensiones en las redes familiares que, al igual que en el apartado de significado de cuidado, están ligadas al uso del castigo físico. E.: "Cuando la mamá no está en la casa, ¿quién te cuida?" EN.: “eh... mi tía y ella me pega" (GF2FPI2). 
En lo que corresponde a la red institucional, se reitera que los niños y las niñas son atendidos y atendidas en instituciones que hacen parte de la Alianza para la Infancia en la ciudad de Medellín; allí reciben de lunes a viernes acompañamiento en alimentación, educación, recreación, entre otros. Estos espacios se constituyen en redes para sus padres y madres, quienes requieren acceder a labores remuneradas. Como lo plantean Esquivel et al. (2011):

El acceso a jardines maternales y jardines de infantes puede ser un factor decisivo en el tiempo que madres y padres dedican al cuidado de los niños y las niñas de hasta 5 años, ya sea directamente, absorbiendo parte del tiempo que, de otro modo, dedicarían al cuidado de sus hijos e hijas, o indirectamente, brindando a padres y madres la posibilidad de acceder a trabajos remunerados (pp. 83-84).

Igualmente, los niños y las niñas consideran que estos espacios representan un apoyo para sus familias. E.: "¿para qué le sirve a tu familia contar con la ayuda de estas personas o sitios donde te cuidan?" EN.: "Para descansar" (GF1FN7). Ahora bien, de forma particular esta red se nombra directamente como un escenario de diversión: E. 2: “¿Qué hacemos acá en el jardín?" EN: "Jugamos con los juguetes." EN.: "¡Con los disfraces!" EN.: "Jugamos balón" (GF2MPI1, GF2FPI2, GF2MPI3). De acuerdo con sus relatos el juego es un componente con el que refieren sentirse satisfechos y felices.

La vinculación a este servicio, donde los niños y las niñas cuentan con espacios de lúdica, socialización y experiencias de formación, complementa las tareas y responsabilidades de sus cuidadores y cuidadoras principales, debido a que sus ocupaciones laborales a veces limitan los tiempos para el cuidado de los niños y las niñas; de ahí que el establecimiento de esta red posibilita generar ingresos económicos $\mathrm{y}$, a su vez, dar respuesta a las necesidades de cuidado de estos y estas. Como lo plantean Betancourt y Escobar (2016): "las instituciones educativas, las organizaciones del Tercer Sector como las redes vecinales y las $\mathrm{ONG}$, se convierten en espacios que además de formación académica deben suplir necesidades de cuidado" (p. 34).
En cuanto a las redes vecinales, es decir, personas externas a la familia que han generado confianza en los cuidadores y cuidadoras principales, en esta investigación se pudo establecer que, en las familias abordadas, esta se usa en menor proporción que las redes familiar e institucional; aunque en los relatos de los niños y las niñas no se precisan las razones de esto. Como elemento en común, los niños y las niñas expresan sentirse satisfechos con esta red, debido a la realización de actividades relacionadas con el juego o a que encuentran pares de edades similares con quienes establecen empatía y comparten actividades que los y las divierten. E.: "Cuando tu mamá lleva a tu abuela al médico y tu abuelo está en el trabajo, ¿quién te cuida?" EN.: "Me voy para la casa de mi amigo" (GF4MPI19).

Como aspecto particular, se evidencian tensiones en tanto que los niños y las niñas, expresan aburrimiento: E.: "Cuándo te cuida un vecino, ¿te gusta?” EN.: "No.” E.: “¿Por qué?" EN.: "Ay, no uno se siente aburrida, sin alientos, no lo dejan salir" (GF1FN11). A su vez, se encontraron tensiones en cuanto a los "mecanismos de cuidado" que se utilizan en esta red, en los que también aparece el castigo físico, lo cual es bastante alarmante. La participación de otras personas en el cuidado de los niños y las niñas concuerda con lo que señalan Atehortúa, Sánchez y Jiménez (2009): "las dificultades para el funcionamiento familiar y el cuidado de sus miembros, se han reforzado las redes de parentesco y de amistad" (p. 129). Esta delegación del cuidado en familiares y personas cercanas se hace considerando, además de los lazos consanguíneos, los vínculos afectivos y la búsqueda de principios morales, éticos, religiosos, familiares y sociales afines, ya que así se generan la seguridad y confianza para garantizar que estos sean transmitidos a la persona cuidada durante el tiempo en el cual se encuentra bajo la protección del tercero y, adicionalmente, se logre mantenerlos en el tiempo y ser transmitidos entre las diferentes generaciones que a futuro podrán ser cuidadoras.

Es así como las redes de apoyo se convierten para los cuidadores y cuidadoras principales en un refuerzo para el cuidado, en tanto favorecen la asistencia a labores remuneradas, diligencias 
$\mathrm{u}$ otros compromisos y, a la vez, permiten que los niños y las niñas conozcan nuevos espacios y personas que les proporcionen el cuidado desde la perspectiva con la que ellos y ellas se encuentran satisfechos.

Aquellos niños y niñas que refieren insatisfacción en el cuidado por parte de la red de apoyo, aluden la presencia del castigo físico; con lo cual es necesario enfatizar en las prácticas formativas de nuestro contexto para que se logre erradicar de ellas la fuerza, la humillación y el desconocimiento de la condición de sujetos de quienes son cuidados.

\section{Conclusiones}

Recurrir a las voces de los niños y las niñas permite ampliar y comprender la noción de cuidado, pues según lo que se evidenció mediante los grupos focales, ellos y ellas en sus narrativas hacen alusión a las dimensiones del mismo, las cuales son abordadas de manera consistente por investigadores e investigadoras, como se mencionó en este texto. Es así como los sentires de los niños y las niñas evocan las tres dimensiones del cuidado (material, económica y afectiva) de forma relacionada y complementaria. Sin embargo, la dimensión afectiva fue la más representativa para ellos y ellas, identificándola en actos como la recreación, el juego, las expresiones de amor y el acompañamiento. Quien incorpora estas prácticas se constituye en el cuidador o cuidadora de su predilección, por cuanto es con quien sienten mayor satisfacción y disfrute.

En este sentido, los niños y las niñas denotan preferencias por un género u otro a partir de las actividades que hombres y mujeres efectúan dentro y fuera del hogar para su cuidado. Esas actividades continúan conexas a lo que culturalmente realiza cada género. En los significados expresados por quienes participaron en los grupos focales, la acción de cuidado trasciende al compartir momentos y posibilitar el fortalecimiento de vínculos afectivos con familiares y otras personas que asumen el acompañamiento cotidiano, idenitificándose con condiciones de bienestar que gratifican a niños y niñas y contribuyen a su crecimiento y desarrollo. En contraposición, se observa en sus relatos cuando de manera explícita nombran el castigo físico, al mostrarse inconformes con esta práctica pues no comprenden el porqué de la misma y la cuestionan como una acción de cuidado.

En la investigación se halló que prevalece el cuidado como una actividad realizada por mujeres, quienes desempeñan actividades relacionadas con el hogar y, al mismo tiempo, aportan en la construcción de estereotipos por parte de las niñas acerca de la feminidad. Según lo anterior, puede decirse que en los relatos de los niños y las niñas las mujeres siguen sobrecargadas de responsabilidades, aun cuando ellos y ellas no lo denoten de esta manera, justamente porque así van asimilando los patrones culturales que todavía son tan imperativos en nuestro medio. La participación de los hombres en actividades de cuidado - principalmente recreativas - resulta significativa para los niños y las niñas. Dicha participación puede aludir a modificaciones al interior de las familias, las cuales no desplazan a la mujer como figura principal en el cuidado, sino que delegan en éstos algunas tareas.

Los arreglos que realizan los cuidadores y cuidadoras principales de la investigación para el cuidado de los niños y las niñas evidenciaron redes de apoyo con las cuales cuentan y, además, las opciones que tienen para acceder a un trabajo remunerado que les permita complementar el cuidado de los niños y las niñas desde la dimensión económica.

\section{Lista de referencias}

Agudelo-Bedoya, M. E., \& Estrada, P. (2011). Fundamentos del construccionismo social y prácticas terapéuticas inspiradas en éstos. (Trabajo de grado de Maestría). Universidad Pontificia Bolivariana, Medellín, Colombia.

Agudelo-Bedoya, M. E., \& Estrada, P. (2013). Terapias narrativas y colaborativa: una mirada con el lente del construccionismo social. Revista Facultad de Trabajo Social, 29, (29), 15-48.

Aguirre, R. (2003). El cuidado infantil en Montevideo. Análisis de los resultados de la encuesta sobre usos del tiempo: 
desigualdades sociales y de género. Montevideo: Unicef Uruguay.

Aguirre, R. (2008). El futuro del cuidado. En I. Arriagada. (Ed.), El futuro de la familia (pp. 23-34). Santiago de Chile: Cepal.

Atehortúa, C., Sánchez, L., \& Jiménez, B. (2009). El conflicto armado afecta todas las esferas: implicaciones del conflicto armado en la comuna 13. Revista de Derecho, (32), 116-138. Recuperado de: http://rcientificas. uninorte.edu.co/index.php/derecho/article/ view/663.

Barrera, A. M., Duque, C., Restrepo, C., Pérez, D., Martínez, Y., Palacios, X., ... Sánchez N. (2015). Aproximación a los significados, a los usos del mercado y al género en la organización social del cuidado de niños $y$ niñas menores de 12 años de grupos familiares en Medellín. (Trabajo de grado de especialización). Universidad Pontificia Bolivariana, Medellín, Colombia.

Betancourt, M. J., \& Escobar, S. C. (2016). Investigación-acción: una reflexión desde la organización social del cuidado de niños y niñas en Cali-Colombia en el marco de la política de 0 a siempre. Derecho y Ciencias Sociales, (14), 26-61. Recuperado de: http://hdl.handle.net/10915/53096.

Cafaro M. A., \& Espasandin, C. M. (2015).

El sistema nacional de cuidados en el

Uruguay: género y cuidados en el foco del debate. Fronteras, (8), 119-132.

Carrasco, C. (2006). La paradoja del cuidado: necesario pero invisible. Revista de Economía Crítica, (5), 39-64.

Esquivel, V., Faur, E., \& Jelin, E. (2011). Las lógicas del cuidado infantil, entre las familias, el Estado y el mercado. Buenos Aires: Ides.

Flores, Á. R. \& Tena, G. O. (2014). Maternalismo $\mathrm{y}$ discursos feministas latinoamericanos sobre el trabajo de cuidados: un tejido en tensión. Íconos. Revista de Ciencias Sociales, (50), 27-42.

Galeano, E. (2014). Diseño de proyectos en investigación cualitativa. Medellín: Universidad Eafit.

Garcés, D., \& Chamorro, L. (2011). Niñas, niños y jóvenes desvinculados del conflicto armado: ¿qué sucede con sus vínculos afectivos y con sus figuras de apego antes y durante su permanencia en los grupos armados? Revista Eleuthera, 5, 36-50. Recuperado de: http://eleuthera.ucaldas. edu.co/downloads/Eleuthera5 5.pdf.

Gómez-Urrutia, V. \& Jiménez-Figueroa, A. (2015). El conflicto trabajo-familia ante los derechos al cuidado de niños y niñas. Revista Latinoamericana de Ciencias Sociales, Niñez y Juventud, 13(1), 137150. doi:10.11600/1692715x.1317190814

Jiménez B. A. \& Torres C. A. (Comps.) (2006). La práctica investigativa en ciencias sociales. Bogotá, D. C.: Universidad Pedagógica Nacional.

Micolta, A., Paz, D., \& Jiménez, Y. (2016, agosto). El cuidado familiar de niños y niñas en contextos de pobreza y violencia. Ponencia presentada en el II Congreso Internacional de Familias y Redes Sociales, Córdoba, Argentina.

Posada-Díaz, A., Gómez-Ramírez, J., \& Ramírez-Gómez, H. (2008). Crianza humanizada: una estrategia para prevenir el maltrato infantil. Acta Pediátrica de México, 29, (5), 295-305. Recuperado de: http://www.medigraphic.com/pdfs/ actpedmex/apm-2008/apm085j.pdf

Salas, J. (2013). La familiarización y desfamiliarización de la crianza y los cuidados de la infancia. Medellín: Fundación Bienestar Humano.

Sánchez, G., \& Palacio, M. C. (2013). Cuidado familiar, orden discursivo hegemónico y contrahegemónico. Revista Latinoamericana de Estudios de Familia, (5), 29-45.

Triana, N., Ávila, L., \& Malagón, A. (2010). Patrones de crianza y cuidado de niños y niñas en Boyacá. Revista Latinoamericana de Ciencias Sociales, Niñez y Juventud, 8, (2), Recuperado de: http:// revistaumanizales.cinde.org.co/index. $\mathrm{php} /$ Revista-Latinoamericana/article/ viewArticle/81.

Zibecchi, C. (2014). Cuidadoras del ámbito comunitario: entre las expectativas de profesionalización y el altruismo. Íconos. Revista de Ciencias Sociales, (50), 129-145. Recuperado de: http://dx.doi. org/10.17141/iconos.50.2014.1433. 